\title{
CORRECTION
}

\section{Author Correction: IMMP2L: a mitochondrial protease suppressing cellular senescence}

\author{
Valentina Sica ${ }^{1,2,3,4}$ and Guido Kroemer ${ }^{1,2,3,4,5,6}$ \\ Cell Research (2018) 28:782; https://doi.org/10.1038/s41422-018-0059-x
}

Correction to: Cell Research 28, 607-608 (2018); https://doi.org/ 10.1038/s41422-018-0051-5; published online 29 May 2018.

We apologize for an error we just found in the paper published online on 29 May 2018. The protein SERPINB4 is mistakenly indicated as SERPIN4B for three times in the main text and once in Fig. 1. The corrected sentences and Fig. 1 are provided below.
It remains to be seen, however, whether transgene-enforced upregulation of IMMP2L or depletion/deletion of its inhibitor SERPINB4 may reduce the manifestations of aging in whole organisms. Moreover, it might be interesting to develop pharmacological inhibitors of the IMMP2L/SERPINB4 interaction to allow newly synthesized IMMP2L to escape from its irreversible blockade by SERPINB4, thus reactivating its function.

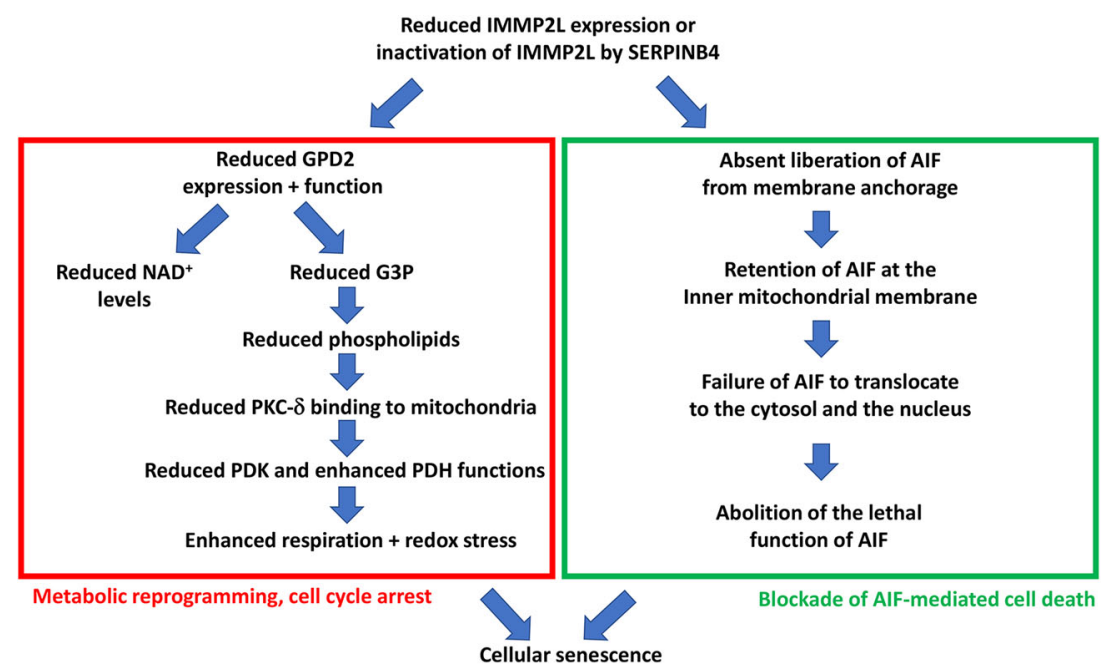

Fig. 1 Schematic overview of the pathway linking IMMP2L to senescence. Inactivation or inhibition of IMMP2L causes the inhibition of the expression and function of GPD2, thereby inducing metabolic reprogramming of the cell. Suppression of IMMP2L also leads to the selective inactivation of the pro-death function of AIF. Together, these alterations cause cellular senescence. The molecular links to the upregulation of CDK inhibitors and the SASP remain to be established. Please ignore this correction and DO NOT add the period

${ }^{1}$ Equipe 11 labelisée par la Lique Nationale contre le Cancer, INSERM U1138, Centre de Recherche des Cordeliers, 75006 Paris, France; ${ }^{2}$ University of Paris Descartes, Sorbonne Paris Cité, 75006 Paris, France; ${ }^{3}$ University of Pierre et Marie Curie, 75006 Paris, France; ${ }^{4}$ Metabolomics and Cell Biology Platforms, GRCC, 94805 Villejuif, France; ${ }^{5}$ Pôle de Biologie, Hôpital Européen Georges Pompidou, 75015 Paris, France and 'Karolinska Institute, Department of Women's and Children's Health, Karolinska University Hospital, 17176 Stockholm, Sweden

Correspondence: Guido Kroemer (kroemer@orange.fr)

Published online: 19 June 2018 